\title{
Correlation between methylenetetrahydrofolate reductase gene polymorphism and oligoasthenospermia and the effects of folic acid supplementation on semen quality
}

\author{
Chong Xie ${ }^{1,2,3 \#}$, Ping Ping ${ }^{4,5 \#}$, Yi Ma ${ }^{4,5 \#}$, Zhengmu Wu ${ }^{1,2,3}$, Xiangfeng Chen ${ }^{4,5,6}$ \\ ${ }^{1}$ Center for Reproductive Medicine, the International Peace Maternity and Child Health Hospital, School of Medicine, Shanghai Jiao Tong \\ University, Shanghai 200030, China; ${ }^{2}$ Shanghai Key laboratory of Embryo Original Diseases, Shanghai 200030, China; ${ }^{3}$ Shanghai Municipal Key \\ Clinical Specialty, Shanghai 200030, China; ${ }^{4}$ Center for Reproductive Medicine, Ren Ji Hospital, School of Medicine, Shanghai Jiao Tong University, \\ Shanghai 200135, China; ${ }^{5}$ Shanghai Key Laboratory for Assisted Reproduction and Reproductive Genetics, Shanghai 200135, China; ${ }^{6}$ Shanghai \\ Human Sperm Bank, Shanghai 200135, China \\ Contributions: (I) Conception and design: C Xie, P Ping, X Chen; (II) Administrative support: C Xie, P Ping, X Chen; (III) Provision of study \\ materials or patients: All authors; (IV) Collection and assembly of data: All authors; (V) Data analysis and interpretation: C Xie, P Ping, X Chen; (VI) \\ Manuscript writing: All authors; (VII) Final approval of manuscript: All authors. \\ \#These authors contributed equally to this work. \\ Correspondence to: Dr. Xiangfeng Chen. Center for Reproductive Medicine, Ren Ji Hospital, School of Medicine, Shanghai Jiao Tong University, \\ Shanghai 200135, China. Email: allanbacon@163.com.
}

Background: The present study investigated the correlation between 5,10-methylenetetrahydrofolate reductase (MTHFR) C677T polymorphism and oligoasthenospermia, as well as the effects of folic acid supplementation on semen quality.

Methods: The present study was a case control study. The PCR-chip assay was applied to analyze the distribution characteristics of the frequencies and genotypes of the MTHFR C677T allele in 167 Han Chinese patients with idiopathic male infertility (including 86 patients with oligospermia and 81 patients with asthenospermia) and in 78 males with normal semen parameters. Moreover, homocysteine (Hcy) levels were assessed for the different groups. Semen quality was measured following three months of folic acid supplementation for the oligospermia and asthenospermia groups.

Results: The cytosine-thymine (CT) genotype (50\% vs. 39.5\%) and the thymine-thymine (TT) genotype $(51.2 \%$ vs. $7.7 \%)$ carriers in the oligospermia group exhibited significantly higher percentages compared with those of the control group. The percentage of the CT genotype carriers in the asthenospermia group was significantly higher compared with that of the control group (59.3\% vs. 50\%), while the frequency of the TT genotype was significantly increased (22.2\% vs. $7.7 \%)$. Furthermore, serum Hcy levels in the oligospermia and asthenospermia groups were significantly higher compared with those of the control group. The data also demonstrated that sperm density increased significantly following three months of folic acid supplementation to patients with oligospermia or asthenospermia. In these patients, the highest increase was noted for the subjects carrying the TT genotype.

Conclusions: The MTHFR C677T mutation and the elevated Hcy levels are important risk factors for the development of oligoasthenospermia. Folic acid supplementation can significantly improve sperm density.

Keywords: Methylenetetrahydrofolate reductase (MTHFR); folic acid; oligospermia; asthenospermia; sperm density

Submitted May 16, 2019. Accepted for publication Nov 05, 2019.

doi: $10.21037 /$ tau.2019.11.17

View this article at: http://dx.doi.org/10.21037/tau.2019.11.17 


\section{Introduction}

Infertility has become a major public health problem. According to the World Health Organization (WHO), it affects approximately $15 \%$ of the couples that are at the age of conceiving a child and approximately $50 \%$ of male subjects (1). Several different causes of male infertility have been proposed with semen abnormalities caused by spermatogenic disorders being the most common. Major semen abnormalities are oligospermia and asthenospermia and consequently oligoasthenospermia is the main disorder that is targeted therapeutically for male infertility $(2,3)$.

The pathogenesis of oligospermia and asthenospermia is very complex and it includes chromosomal abnormalities, obstruction of spermatic ducts, genital tract infections, structural abnormalities in the sperm and semen abnormalities (4). In recent years, a large number of chromosomal malformations and individual gene mutations that can lead to male infertility have been identified (5). Oligoasthenospermia is caused by genetic factors in approximately $21-29 \%$ patients. Chromosomal abnormalities account for only a small number of these factors, while the involvement of pathogenic genes remains unclear for the majority of the patients (6). Based on knockout mouse studies, 388 genes have been associated with sperm abnormalities, leading to oligospermia and even azoospermia in mice (7), which in turn suggests that genetic mutations are an important cause for sperm dysfunction. In recent years, different studies have demonstrated that DNA methylation abnormalities and oxidative stress in sperms are important factors involved in male infertility (8-11). Folic acid is an important methyl donor in the human body and therefore genes associated with the folic acid circulation pathway have become the research focus for male infertility (12-14). 5,10-methylenetetrahydrofolate is transformed to 5 -methyltetrahydrofolate by 5,10-methylenetetrahydrofolate reductase (MTHFR) in the folic acid metabolism pathway (15). The gene that encodes this enzyme is considered a significant contributor to diseases associated with folic acid metabolism, since mutation of $\mathrm{C}$ to $\mathrm{T}$ in the 677 locus of this gene can cause a decrease in the enzymatic activity of MTHFR. This decrease leads to various disorders in the folic acid metabolic pathway, including reduction in methyl donors and an increase in the homocysteine (Hcy) levels $(16,17)$. Therefore, this gene has become a predisposing factor for male infertility $(14,17-20)$. The present prospective study analyzed the effect of the MTHFR polymorphism on folic acid supplementation in 167 cases with idiopathic male infertility. The association between MTHFR polymorphisms and male infertility was explored in a Chinese Han population. Moreover, the effects of folic acid supplementation on sperm quality were investigated.

\section{Methods}

\section{Study subjects}

A total of 167 patients with idiopathic male infertility, including 86 with oligospermia and 81 with asthenospermia, were enrolled in the Department of Reproductive Medicine at the affiliated International Peace Maternal and Child Health Hospital of the Shanghai Jiao Tong University School of Medicine between August 2017 and May 2018. The patients had an age range of 22 to 44 years and the average age was $32.6 \pm 10.3$ years. All the patients were married for more than two years and had normal sexual life. The patients exhibited no unhealthy habits, such as smoking, alcohol drinking or drug abuse. Moreover, the patients were not exposed to harmful substances, such as radiation and chemical reagents at any time during their life. Their partners also had regular menstruation, normal gynecological examination tests, and they did not use any method of contraception to avoid pregnancy. Moreover, chromosomal karyotype abnormalities, Y chromosome microdeletion of sperm factor azoospermia factor (AZF), testicular and epididymal inflammations, varicocele and histories of juvenile mumps and cryptorchidism were excluded. The diagnostic criteria of oligospermia and asthenospermia were assessed according to the fifth edition of the WHO. Two consecutive semen examinations indicated that sperm density was $<15 \times 10^{6} / \mathrm{mL}$, sperm progressive motility $(a+b$ grade) was $<32 \%$ and the rate of normal sperm morphology was $\geq 4 \%$ in the oligospermia group. In contrast to these findings, in the asthenospermia group, sperm progressive motility ( $\mathrm{a}+\mathrm{b}$ grade) was $<32 \%$, sperm density was $\geq 15 \times 10^{6} / \mathrm{mL}$ and the rate of normal sperm morphology was $\geq 4 \%$. A total of 78 cases were present in the control group from the Shanghai Human Sperm Bank Department of Reproductive Medicine at the affiliated Renji Hospital Shanghai Jiao Tong University School of Medicine. The subjects were between the age of 25 and 38 years and the average age was $32.3 \pm 11.2$ years. The patients in the control group presented with natural fertility history, and their semen parameters were normal.

The present study was approved by the Ethics 
Committee of the International Peace Maternal and Child Health Hospital of the Shanghai Jiao Tong University (No. 17-0012).

\section{Detection of MTHFR C677T polymorphism}

A total volume of $2 \mathrm{~mL}$ venous blood was extracted and placed in EDTA anticoagulant blood collection tubes, whereas $200 \mu \mathrm{L}$ was used for genomic DNA extraction. Genomic DNA was extracted using the Blood Genome Extraction Kit (Shanghai BaiO Technology Co., Ltd., Shanghai, China) following the manufacturer's instructions. The PCR amplification was conducted as follows: the MTHFR (C677T) gene detection kit (Shanghai BaiO Technology Co., Ltd.) was used for PCR amplification and sequencing. The reaction contained $5 \mu \mathrm{L}$ reaction solution A, $18 \mu \mathrm{L}$ amplification solution and $2 \mu \mathrm{L}$ genomic DNA of the sample. The amplification program used was the WarmStart and the PCR amplification conditions were as follows: $94{ }^{\circ} \mathrm{C}$ for $5 \mathrm{~min}, 94^{\circ} \mathrm{C}$ for $30 \mathrm{~s}, 56^{\circ} \mathrm{C}$ for $40 \mathrm{~s}$ and $72{ }^{\circ} \mathrm{C}$ for $40 \mathrm{~s}$. The program included 35 cycles and the last cycle was extended for $5 \mathrm{~min}$. A total of $10 \mu \mathrm{L}$ PCR reaction product was subjected to hybridization. Patient genotyping was performed by the biochip reader.

\section{Determination of serum Hcy}

Fresh blood samples were collected and centrifuged at $3,000 \mathrm{r} / \mathrm{min}$ for 6 to $10 \mathrm{~min}$. The serum was separated and serum Hcy levels were measured using a Hitachi 7180 automated biochemical analyzer. A two-point rate assay was used and the main wavelength was set at $340 \mathrm{~nm}$. The secondary wavelength was set at $405 \mathrm{~nm}$. The serum was reacted at $37{ }^{\circ} \mathrm{C}$ for $10 \mathrm{~min}$. A two-point linear correction was used as the calibration method.

\section{Treatment of folic acid supplementation}

Treatment of folic acid supplementation was performed for all patients with oligospermia and asthenospermia. A dose of $400 \mu \mathrm{g}$ was administered twice per day for three months. The semen quality parameters were reviewed following three months for all patients and the comparisons were performed with the parameters obtained prior to the treatment.

\section{Statistical analysis}

The categorical data related to the distribution and frequency of the gene polymorphisms were assessed by the $\chi^{2}$ test in order to analyze the differences between groups. The continuous data related to Hcy levels and sperm density were represented by the mean \pm standard deviation. The data that satisfied the normal distribution and the variance homogeneity were analyzed by the $T$ test or the variance analysis in order to assess differences between the groups. In any other case nonparametric tests were performed. Statistical analysis was performed using the SPSS 22.0 software. A $\mathrm{P}$ value less than $0.05(\mathrm{P}<0.05)$ was considered for significant differences.

\section{Results}

\section{Distribution of MTHFR polymorphism in each group}

A total of 86 patients with oligospermia, 81 patients with asthenospermia and 78 patients with normal fertility were enrolled in the present study. The three genotypes of cytosine-cytosine (CC), cytosine-thymine (CT) and thymine-thymine (TT) were noted in the 677 locus of the MTHFR gene in the Chinese population. The distribution frequencies of the three genotypes, along with the allele frequencies are shown in Table 1. The Chi-squared test was used to analyze the Hardy-Weinberg equilibrium between different case groups and the control group. The results indicated that the actual and the theoretical frequencies were consistent among the three groups $(\mathrm{P}>0.05)$, suggesting that the frequency of this sample was in accordance with the Hardy-Weinberg equilibrium and that the selected samples were representative of the population.

The genotype frequencies of the MTHFR C677T locus was significantly different in the three groups. The CT ( $39.5 \%$ vs. $50 \%, \mathrm{P}=0.0046$, OR $=3.60,95 \% \mathrm{CI}$ was $1.46-$ $8.84)$ and TT $\left(51.2 \%\right.$ vs. $7.7 \%, \mathrm{P}=5.08 \times 10^{-11}$, OR $=30.25$, 95\% CI was 9.57-95.63) genotypes in the oligospermia group were significantly higher compared with those of the control group. The mutant allele $\mathrm{T}$ was a risk factor for oligospermia $\left(70.9 \%\right.$ vs. $32.7 \%, \mathrm{P}=4.29 \times 10^{-12}$, OR $=5.02$, $95 \%$ CI was in the range of 3.14-8.03). The MTHFR genotype distribution in the asthenospermia group was significantly different compared with that of the control group. The number of patients with the CT genotype 
Table 1 Comparison between frequencies of different MTHFR C677T genotypes and alleles in the oligospermia, asthenospermia and control groups

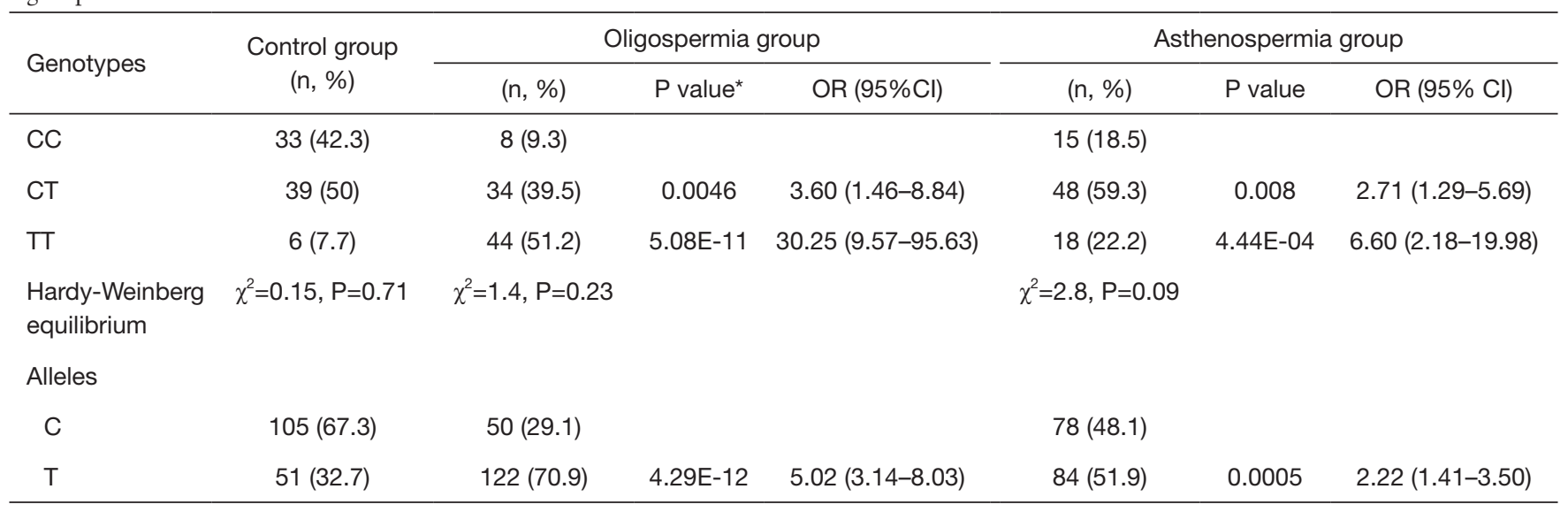

*, $\mathrm{P}<0.01$. MTHFR, methylenetetrahydrofolate reductase; CC, cytosine-cytosine; CT, cytosine-thymine; TT, thymine-thymine; C, cytosine; $\mathrm{T}$, thymine.

Table 2 Comparison of serum homocysteine concentrations $(\mu \mathrm{mol} / \mathrm{mL})$ in patients with oligospermia, asthenospermia and control groups

\begin{tabular}{|c|c|c|c|c|}
\hline Groups (number of cases) & \multicolumn{3}{|c|}{ Genotypes } & Mean concentration \\
\hline Oligospermia group $(n=86)$ & $9.1 \pm 4.3$ & $20.6 \pm 10.7$ & $33.9 \pm 15.5$ & $26.3 \pm 15.5^{\star}$ \\
\hline Asthenospermia group $(n=81)$ & $8.6 \pm 3.8$ & $14.3 \pm 6.4$ & $20.6 \pm 10.1$ & $14.6 \pm 8.0^{\star}$ \\
\hline Control group $(n=78)$ & $8.4 \pm 4.6$ & $12.3 \pm 4.4$ & $20.3 \pm 7.2$ & $11.3 \pm 5.7$ \\
\hline
\end{tabular}

${ }^{*}, \mathrm{P}<0.01$. CC, cytosine-cytosine; CT, cytosine-thymine; T, thymine-thymine.

was significantly higher compared with that of the control group $(59.3 \%$ vs. $50 \%, \mathrm{P}=0.008$, OR $=2.71,95 \% \mathrm{CI}$ was in the range of 1.29-5.69), while the frequencies of the TT genotype were also significantly increased $(22.2 \%$ vs. $7.7 \%$, $\mathrm{P}=4.44 \times 10^{-4}, \mathrm{OR}=6.60,95 \% \mathrm{CI}$ was $\left.2.18-19.98\right)$. The mutant allele was a risk factor for asthenospermia $(51.9 \%$ vs. $32.7 \%, \mathrm{P}=0.0005$, $\mathrm{OR}=2.22,95 \% \mathrm{CI}$ was in the range of $1.41-3.50)$.

\section{Comparison of serum Hcy levels in each group}

Serum Hcy exhibited the highest levels $(26.3 \mu \mathrm{mol} / \mathrm{mL})$ in the oligospermia group, followed by the asthenospermia group $(14.6 \mu \mathrm{mol} / \mathrm{mL})$, while it was the lowest in the control group $(11.3 \mu \mathrm{mol} / \mathrm{mL})$. The serum Hcy levels in the oligospermia group were significantly higher compared with those of the asthenospermia $(\mathrm{P}<0.01)$ and the control groups $(\mathrm{P}<0.01)$ (Table 2). The MTHFR gene grouping demonstrated that the Hcy concentration was the lowest in the CC genotype, followed by the CT genotype, while it was the highest in the TT genotype. The Hcy levels in the mutant patients of the oligospermia group were significantly higher compared with those of the control group (CT: 20.6 vs. $12.3 \mu \mathrm{mol} / \mathrm{mL}, \mathrm{P}<0.01$, TT: 33.9 vs. $20.3 \mu \mathrm{mol} / \mathrm{mL}$, $\mathrm{P}<0.01)$.

\section{Comparison of the semen parameters following folic acid supplementation in each group}

Prior to folic acid supplementation, no significant differences were noted in the sperm density among the different genotypes of the MTHFRC677T locus in the oligospermia group (CC: $7.83 \times 10^{6} / \mathrm{mL}$, CT: $7.31 \times 10^{6} / \mathrm{mL}$, TT: $6.52 \times 10^{6} / \mathrm{mL}$ ). Following three months of folic acid supplementation, an increase in the percentages of the three genotypes was noted, while the increase in the CT and TT genotypes was the most significant. The CT genotype was elevated to $57.5 \%\left(7.31 \times 10^{6} / \mathrm{mL}\right.$ vs. $11.52 \times 10^{6} / \mathrm{mL}$, $\mathrm{P}<0.01)$, whereas the TT genotype was increased to $134.8 \%$ $\left(6.52 \times 10^{6} / \mathrm{mL}\right.$ vs. $\left.15.31 \times 10^{6} / \mathrm{mL}, \mathrm{P}<0.01\right)$. However, the sperm progressive motility was not significantly altered (Table 3, Figure 1). 
Table 3 Comparison of semen quality parameters before and after folic acid supplementation in the oligospermia group

\begin{tabular}{|c|c|c|c|}
\hline Groups & $\mathrm{CC}$ & CT & $\mathrm{TT}$ \\
\hline Sperm density after treatment & $8.24 \times 10^{6} / \mathrm{mL}$ & $11.52 \times 10^{6} / \mathrm{mL}$ & $15.31 \times 10^{6} / \mathrm{mL}$ \\
\hline Ratio of sperm progressive motility before treatment & $41.5 \%$ & $37.3 \%$ & $43.6 \%$ \\
\hline Ratio of sperm progressive motility after treatment & $39.8 \%$ & $40.2 \%$ & $45.1 \%$ \\
\hline
\end{tabular}

$\mathrm{CC}$, cytosine-cytosine; CT, cytosine-thymine; TT, thymine-thymine.

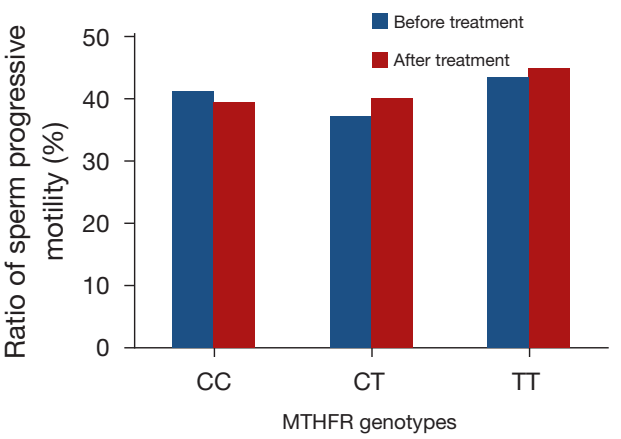

Figure 1 Ratio of sperm progressive motility before and after folic acid supplementation in the oligospermia group. CC, cytosinecytosine; CT, cytosine-thymine; TT, thymine-thymine.

The comparison of the semen quality parameters group prior to and following folic acid supplementation in the asthenospermia subjects is shown in Table 4 and Figure 2. Sub-grouping was performed based on different genotype compositions, which demonstrated that the sperm density of the TT genotype was increased significantly following treatment compared with that noted prior to the treatment. The sperm density of the CT and CC genotypes exhibited no significant change. The ratios of sperm progressive motility of the CT, TT and CC genotypes did not change significantly prior to and following treatment.

\section{Discussion}

MTHFR is a key enzyme involved in the metabolism of folic acid and methionine and catalyses the reduction of 5,10-methylenetetrahydrofolate to 5-methyltetrahydrofolate. This form is the main folid acid bioactive form in vivo, which has two biological functions: (I) it acts as a carrier of one-carbon unit in vivo and is involved in the synthesis of purines and pyrimidines by providing methyl groups for DNA, RNA and proteins, thus affecting normal DNA metabolism; (II) it acts as a methyl donor. 5-methyltetrahydrofolate is the substrate required for Hcy remethylation catalyzed by the enzyme methionine synthase. The end product is methionine, which results in the maintenance of a normal level of plasma Hcy (21).

Previous studies demonstrated that the MTHFR gene exhibited multiple single nucleotide polymorphisms (SNPs). Accordingly, the mutation at the C677T locus has been extensively studied. The replacement of cytosine (C) at the 677 locus by thymine (T) leads to an amino acid misalignment. Alanine, which is present in the catalytic region, is replaced by valine, thus leading to decreased heat tolerance and MTHFR activity. Chen et al. (22) reported that expression of MTHFR in the testes of adult mice was significantly higher compared with other tissues, such as liver and brain, suggesting its relevance in the process of spermatogenesis. Other studies demonstrated that following knockout of the MTHFR gene, mice can develop spermatogenesis disorders and loss of reproductive functions, which further confirms the relevance of the MTHFR gene in the male reproductive systems (23). Currently, a vast number of studies have focused on the association of the C677T locus between MTHFR polymorphisms and male infertility.

A total of 86 patients with oligospermia, 81 patients with asthenospermia and 78 healthy subjects were enrolled in the present study. PCR-chip assay was used to perform genotyping for MTHFR C677T. The obtained results indicated three genotypes in the Chinese population, including CC, CT and TT. Moreover, the frequencies satisfied the Hardy-Weinberg equilibrium in the three groups, which suggested that the selected samples were representative of the genotypes examined. The distribution frequencies of the MTHFR C677T were significantly different in the three groups, and the number of patients with mutations in the CT and TT genotypes in the oligospermia and asthenospermia groups was significantly higher compared with that of the control group, which was consistent with the results obtained by Qiu et al. who 
Table 4 Comparison of semen quality parameters before and after folic acid supplementation in the asthenospermia group

\begin{tabular}{|c|c|c|c|}
\hline Groups & $\mathrm{CC}$ & CT & $\mathrm{TT}$ \\
\hline Sperm density after treatment & $31.04 \times 10^{6} / \mathrm{mL}$ & $41.02 \times 10^{6} / \mathrm{mL}$ & $41.91 \times 10^{6} / \mathrm{mL}$ \\
\hline Ratio of sperm progressive motility before treatment & $19.5 \%$ & $18.3 \%$ & $13.2 \%$ \\
\hline Ratio of sperm progressive motility after treatment & $21.7 \%$ & $16.1 \%$ & $19.9 \%$ \\
\hline
\end{tabular}

CC, cytosine-cytosine; CT, cytosine-thymine; TT, thymine-thymine.

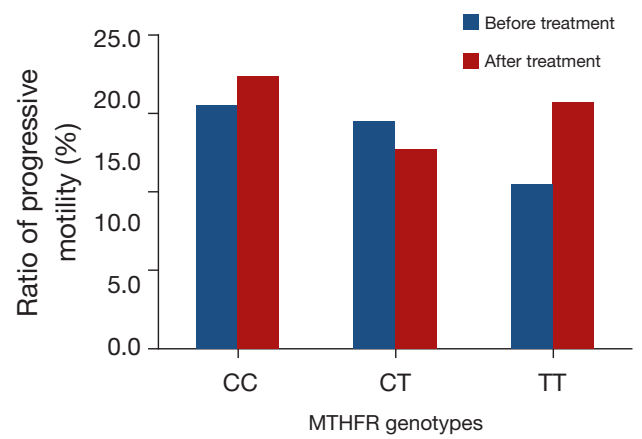

Figure 2 Ratio of sperm progressive motility before and after folic acid supplementation in the asthenospermia group. CC, cytosinecytosine; CT, cytosine-thymine; TT, thymine-thymine.

examined these genotypes in a Han population in Ningxia, China (24). This suggested that the MTHFR C677T mutation may be the predisposing gene for male infertility in China. In a study conducted in Italy, Paracchini et al. (25) demonstrated that the risk factor for infertility was increased within one year in patients with the TT genotype in the MTHFR C677T(OR $=3.7,95 \%$ CI was in the range of 1.4-10.4) locus, suggesting that the MTHFR C677T mutation may also be a risk factor for male infertility in Western countries.

Serum Hcy levels were also measured for the infertility population and the results revealed that Hcy levels were significantly higher in the asthenospermia group compared with those of the control group. Previous studies have shown that the MTHFR polymorphism may cause male infertility via hyperhomocysteinemia (26). Hcy is metabolized via two major pathways in the body as follows: production of methionine by remethylation and production of cystathionine by sulfide transformation. The methyl group was provided by 5 -methyltetrahydrofolate following remethylation of the Hcy. MTHFR is the key enzyme that catalyzes the transformation of 5,10-methylenetetrahydrofolate to 5-methyltetrahydrofolate. Previous studies have suggested that the decrease in MTHFR activity can limit the transformation of Hcy to methionine in vivo, leading to elevated serum Hcy levels (27). High Hcy levels exhibit considerable cytotoxicity and facilitate the oxidation of thiols generating high levels of oxygen free radicals in the cells. This process leads to a decrease in sperm methylation and an increase in DNA fragmentation (9). Moreover, it can also cause damage to sperm mitochondria and nuclear DNA, eventually damaging sperm function (8). Certain studies also have suggested that Hcy can inhibit glutathione synthesis, disrupt the dynamic balance of the production and clearance of active oxygen and consequently affect sperm function $(28,29)$. Other studies have suggested that hyperhomocysteinemia can cause premature arteriosclerosis of blood vessels in the testes, affecting blood supply and leading to spermatogenic dysfunction (30).

In the present study folic acid supplementation was administered for three months to the patients and the results indicated that sperm density was significantly elevated in patients with oligospermia. Among these patients with the MTHFR CT and TT genotypes, the parameter sperm density exhibit highly significant elevation. The patients with the MTHFR TT genotype in the asthenospermia group revealed significantly increased sperm density. Nevertheless, the observed increase was not significant in patients with the CC and CT genotypes, which may be related to the presence of low Hcy levels in the asthenospermia group prior to folic acid supplementation. The MTHFR C677T mutation was proven to be an important factor for the increase of Hcy levels, while folic acid is not an antioxidant that can reduce Hcy levels in the body. Consequently, folic acid can reverse the increase in Hcy levels induced by the MTHFR gene mutation, suggesting that folic acid supplementation was notably important in MTHFR gene mutation. The ratio of sperm progressive motility also did not change significantly prior to and following folic acid supplementation, suggesting that the decrease in the 
progressive motility of infertile patients was not associated with MTHFR gene mutation and folic acid supplementation and that this effect was possibly induced by other causes.

In summary, the present study demonstrated that MTHFR C677T mutation may be a potential risk factor for oligospermia and asthenospermia in Chinese male subjects and that folic acid supplementation could significantly improve sperm density in patients with mutant genes. However, previous studies that examined the association between MTHFR gene polymorphisms and male infertility had shown inconsistent results in China and Western countries. No association was noted between the MTHFR polymorphism and male infertility in Spanish and Polish populations $(12,31)$, which could be explained by the differences in genotype frequencies between ethnicity, as well as the differences in environmental and nutritional factors. These results may also be due to the sample size and due to the different criteria used for the selection of subjects. The accuracy or diversity of the relevant data should be further confirmed by multi-center studies with larger sample sizes.

\section{Acknowledgments}

Funding: This study was supported by the National Natural Science Foundation of China (No. 81671511 to P Ping, 81871199 and 81501310 to Y Ma).

\section{Footnote}

Conflicts of Interest: The authors have no conflicts of interest to declare.

Ethical Statement: The authors are accountable for all aspects of the work in ensuring that questions related to the accuracy or integrity of any part of the work are appropriately investigated and resolved. The present study was approved by the Ethics Committee of the International Peace Maternal and Child Health Hospital of the Shanghai Jiao Tong University (No. 17-0012).

\section{References}

1. Li B, Lan F. Genetic polymorphism and male infertility with sperm specific expressed genes. Zhonghua Nan Ke Xue 2008;14:360-3.

2. Fisher JR, Hammarberg K. Psychological and social aspects of infertility in men: an overview of the evidence and implications for psychologically informed clinical care and future research. Asian J Androl 2012;14:121-9.

3. Winters BR, Walsh TJ. The epidemiology of male infertility. Urol Clin North Am 2014, 41:195-204.

4. Kamel RM. Management of the infertile couple: an evidence-based protocol. Reprod Biol Endocrinol 2010;8:21.

5. Mitchell MJ, Metzler-Guillemain C, Toure A, et al. Single gene defects leading to sperm quantitative anomalies. Clin Genet 2017;91:208-16.

6. Hamada AJ, Esteves SC, Agarwal A. A comprehensive review of genetics and genetic testing in azoospermia. Clinics (Sao Paulo) 2013;68 Suppl 1:39-60.

7. Massart A, Lissens W, Tournaye H, et al. Genetic causes of spermatogenic failure. Asian J Androl 2012;14:40-8.

8. Agarwal A, Makker K, Sharma R. Clinical relevance of oxidative stress in male factor infertility: an update. Am J Reprod Immunol 2008;59:2-11.

9. Tunc O, Tremellen K. Oxidative DNA damage impairs global sperm DNA methylation in infertile men. J Assist Reprod Genet 2009;26:537-44.

10. Castillo J, Estanyol JM, Ballesca JL, et al. Human sperm chromatin epigenetic potential: genomics, proteomics, and male infertility. Asian J Androl 2015;17:601-9.

11. Ko EY, Sabanegh ES Jr, Agarwal A. Male infertility testing: reactive oxygen species and antioxidant capacity. Fertil Steril 2014;102:1518-27.

12. Kurzawski M, Wajda A, Malinowski D, et al. Association study of folate-related enzymes (MTHFR, MTR, MTRR) genetic variants with non-obstructive male infertility in a Polish population. Genet Mol Biol 2015;38:42-7.

13. Gava MM, Kayaki EA, Bianco B, et al. Polymorphisms in folate-related enzyme genes in idiopathic infertile Brazilian men. Reprod Sci 2011;18:1267-72.

14. Liu K, Zhao R, Shen M, et al. Role of genetic mutations in folate-related enzyme genes on Male Infertility. Sci Rep 2015;5:15548.

15. Hiraoka M, Kagawa Y. Genetic polymorphisms and folate status. Congenit Anom (Kyoto) 2017;57:142-9.

16. Frosst $\mathrm{P}, \mathrm{Blom} \mathrm{HJ}$, Milos R, et al. A candidate genetic risk factor for vascular disease: a common mutation in methylenetetrahydrofolate reductase. Nat Genet 1995;10:111-3.

17. Bezold G, Lange M, Peter RU. Homozygous methylenetetrahydrofolate reductase C677T mutation and male infertility. N Engl J Med 2001;344:1172-3.

18. Gong M, Dong W, He T, et al. MTHFR 677C>T polymorphism increases the male infertility risk: a meta- 
analysis involving 26 studies. PloS One 2015;10:e0121147.

19. $\mathrm{Wu} W$, Shen O, Qin Y, et al. Idiopathic male infertility is strongly associated with aberrant promoter methylation of methylenetetrahydrofolate reductase (MTHFR). PloS One 2010;5:e13884.

20. Gupta N, Sarkar S, David A, et al. Significant impact of the MTHFR polymorphisms and haplotypes on male infertility risk. PloS One 2013;8:e69180.

21. Guo KM, Tian RH, Wang HL. Advances in the Relationship between MTHFR Gene Polymorphism and Infertility. Zhonghua Nan Ke Xue 2016;22:171-4.

22. Chen Z, Karaplis AC, Ackerman SL, et al. Mice deficient in methylenetetrahydrofolate reductase exhibit hyperhomocysteinemia and decreased methylation capacity, with neuropathology and aortic lipid deposition. Hum Mol Genet 2001;10:433-43.

23. Kelly TL, Neaga OR, Schwahn BC, et al. Infertility in 5,10-methylenetetrahydrofolate reductase (MTHFR)deficient male mice is partially alleviated by lifetime dietary betaine supplementation. Biol Reprod 2005;72:667-77.

24. Qiu X, Hu X, Li Y, et al. Correlative analysis of C677T polymorphism of MTHFR gene in Han nationality in Ningxia and males and severe oligospermia. Journal of Ningxia Medical University 2011;33:625-8.

25. Paracchini V, Garte S, Taioli E. MTHFR C677T polymorphism, GSTM1 deletion and male infertility: a possible suggestion of a gene-gene interaction? Biomarkers
2006;11:53-60.

26. Liu L, Qian W. Progress in the Research of Polymorphisms in the Methylenetetrahydrofolate Reductase and Methionine Synthase Genes with Male Infertility. J Int Reprod Health/ Fam Plan 2011;30:222-5,39.

27. Han IB, Kim OJ, Ahn JY, et al. Association of methylenetetrahydrofolate reductase (MTHFR 677C>T and $1298 \mathrm{~A}>\mathrm{C}$ ) polymorphisms and haplotypes with silent brain infarction and homocysteine levels in a Korean population. Yonsei Med J 2010;51:253-60.

28. Aitken RJ, Flanagan HM, Connaughton H, et al. Involvement of homocysteine, homocysteine thiolactone, and paraoxonase type $1(\mathrm{PON}-1)$ in the etiology of defective human sperm function. Andrology 2016;4:345-60.

29. Kralikova M, Crha I, Huser M, et al. The intracellular concentration of homocysteine and related thiols is negatively correlated to sperm quality after highly effective method of sperm lysis. Andrologia 2017. doi: 10.1111/ and.12702.

30. Rossato M. A genetic polymorphism and male fertility. Fertil Steril 2004;81:1429-30; author reply 30-1.

31. Camprubi C, Pladevall M, Grossmann M, et al. Lack of association of MTHFR rs1801133 polymorphism and CTCFL mutations with sperm methylation errors in infertile patients. J Assist Reprod Genet 2013;30:1125-31.
Cite this article as: Xie C, Ping P, Ma Y, Wu Z, Chen X. Correlation between methylenetetrahydrofolate reductase gene polymorphism and oligoasthenospermia and the effects of folic acid supplementation on semen quality. Transl Androl Urol 2019;8(6):678-685. doi: 10.21037/tau.2019.11.17 